\title{
EPIDEMIOLOGY OF COLORECTAL CANCER
}

\author{
Vladimír Janout, Helena Kollárová
}

Department of Preventive Medicine, Faculty of Medicine, Palacký University, Hněvotínská 3, 77515 Olomouc

Received February 13, 2001

Key words: Colorectal cancer / Epidemiology / Risk factors

This review article presents colorectal cancer epidemiology, describing main epidemiologic characteristics and the influence of environmental and life style risk factors. The most important ideas for primary prevention are listed at the end of the article.

\section{INTRODUCTION}

Colorectal cancer is the fourth most common cancer in the world with approximately 875,000 new cases per year worldwide (WHO 1996), accounting for $8.5 \%$ of all new cancers.

High risk areas are considered North America, Europe and Australia.The developed world accounts for over $63 \%$ of all cases. Colon cancer is more common in developed countries exhibiting Westernized lifestyle practices.

There are different gender distributions with similar incidences between the sexes in the colon and a male predominance for rectum cancer (20-50\% higher).

The incidence is determined largely by environmental exposure. The evidence comes from studies of migrants when rates in migrants from low to high risk countries tend to increase to the rates of the host countries (within the first or second generation or even as early as within the migrating generation itself). Geographic location of the country of origin, age at migration, duration of residence in the adoptive country and extent of cultural assimilation influence the level and speed of an increase.

There are some examples available, such as the mortality of Japanese Americans being significantly higher than that among the Japanese in Japan and, by the early 1970s, the Japanese in Hawaii had a mortality similar to that of U.S. whites. Also in Chinese migrating to the USA, mainly from one province in China, the mortality rate among the first generation of migrants was 2.7-5.6 times higher than found in that province. Another example describes the incidence differences in Israeli Jews according to place of birth:

- in Europe or America (22.5/100 000),

- in Israel (18.1/100 000),

- in Africa or Asia (13.2/100 000),

- as well as in Israeli Non-Jews (4.6/100 000).

Apart from migration there are some other factors influencing differences in incidence of colorectal cancer. One of them, for example, is urbanization. The incidence is consistently higher among urban residents. This urban excess of incidence is more apparent for men than women and for colon cancer than for rectal cancer. Current residence in an urban area is a stronger predictor of risk than is place of birth (risk for persons born and currently residing in rural areas is $30 \%$ less than that for urban residents).
Another factor is socioeconomic status. Mortality from cancer was positively correlated with indices of socioeconomic status like

- income level

- education

- access to and quality of medical care (survival).

At present, in contrast to the international incidence, patterns of upper digestive tract squamous cell carcinomas and gastric adenocarcinomas, which demonstrate significant inverse correlations with socioeconomic factors and the current RR of colorectal adenocarcinomas are not uniformly associated with social and economic advancement.

\section{INCIDENCE}

In the last decades Europe has seen a widespread increase in the incidence of colorectal cancer, more marked in Eastern than in Western populations. The incidence in the Czech Republic is one of the highest in Europe, particularly the incidence of rectal cancer is the highest in Europe in both male and female populations (fig. 1, 2, 3, 4).

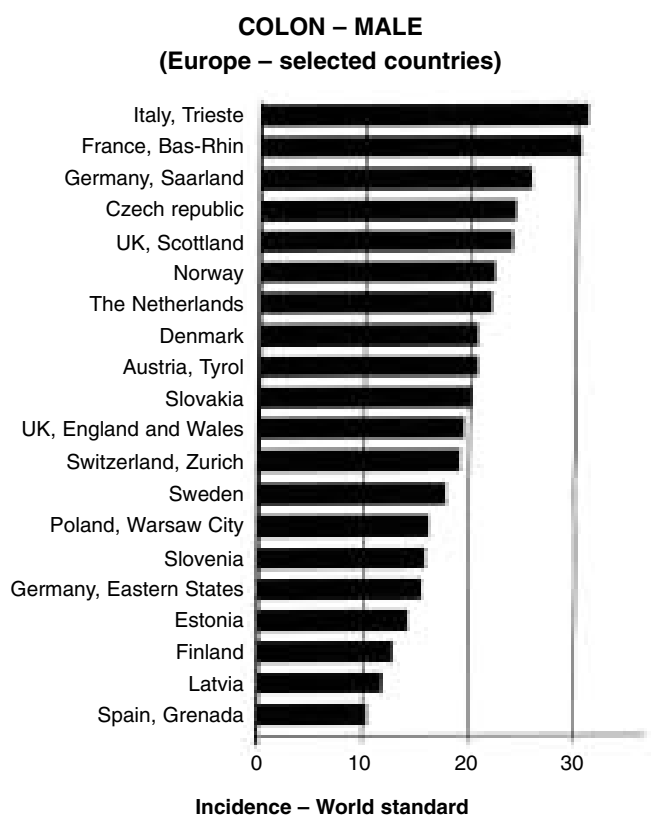

Fig. 1.: Age-standardized incidence of colon cancer, male population, 1988-1992 


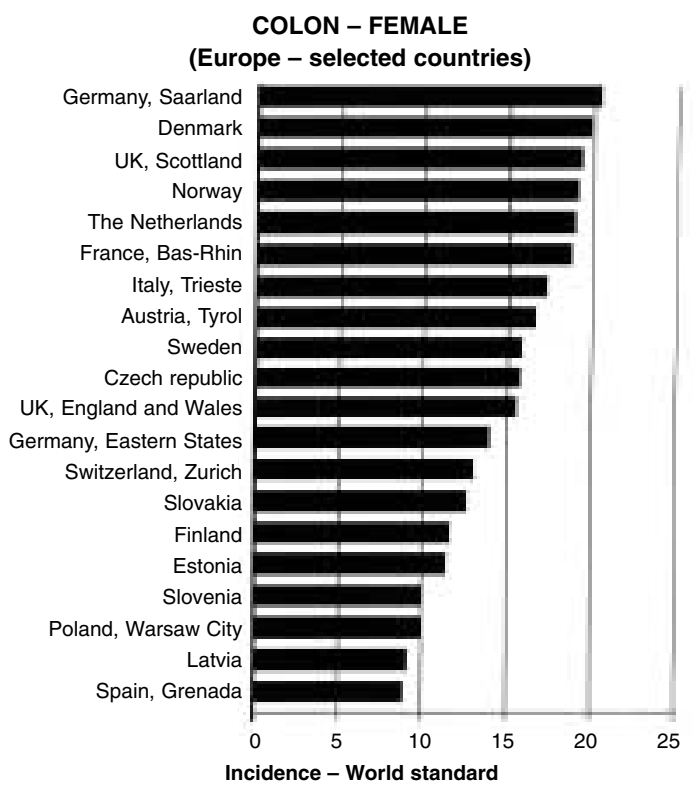

Fig. 2.: Age-standardized incidence of colon cancer, female population, 1988-1992

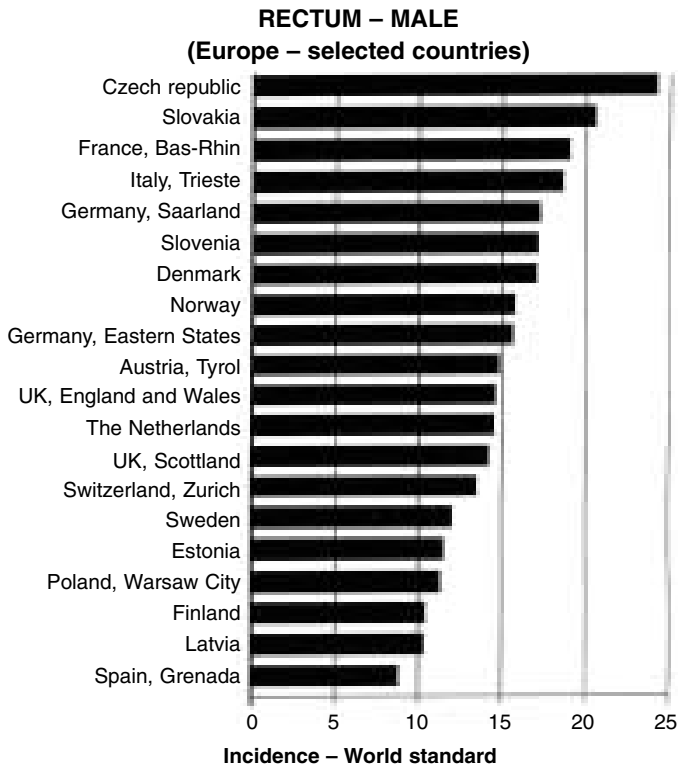

Fig. 3.: Age-standardized incidence of rectal cancer, male population, 1988-1992

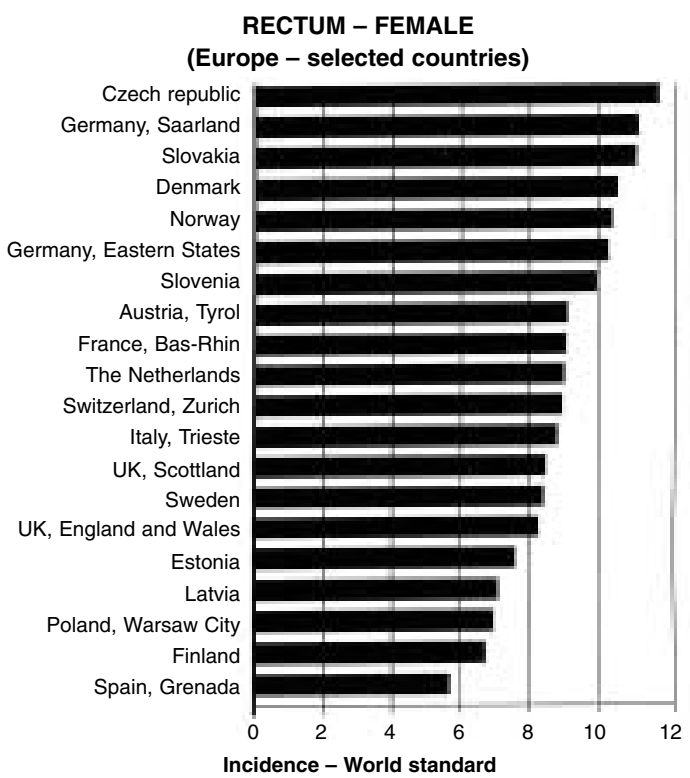

Fig. 4.: Age-standardized incidence of rectal cancer, female population, 1988-1992

\section{MORTALITY}

Worldwide mortality attributable to colorectal cancer in 1996 was estimated at 500,000 people, that is $7.2 \%$ of all cancer deaths.

By contrast to the incidence, there has been a decline in mortality in Europe, particularly in EC countries and other Western European countries. Mortality trends show a marked geographical gradient, starting in the North in the1960's (UK and Denmark), affecting Southern and Western Europe in the 1970's ( the rest of the EC countries) and Greece after 1980.

In the Czech Republic, Hungary, Poland and Yugoslavia, mortality has been increasing $11-15 \%$ in males, $5-8 \%$ in females every 5 years (fig. 5,6 )

\section{COLON AND RECTUM (ICD-9 153-154)}

EUROPE (non-EC), MORTALITY

Percentage change per five-year period, 1975-1988

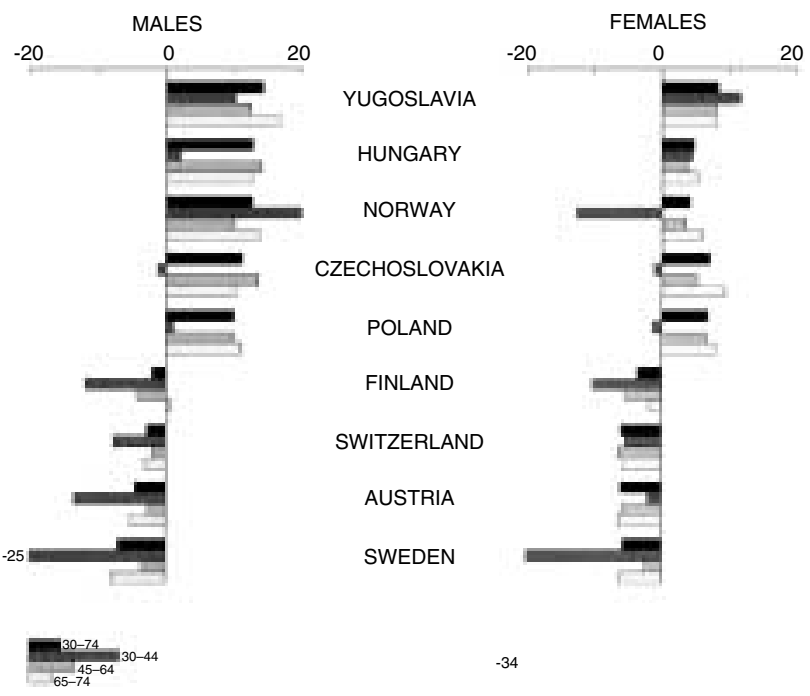

Fig. 5.: Colorectal cancer age-specific mortality rates, estimated mean percentage change per five-year period over the period 1975-1988 - non EC countries

\section{COLON AND RECTUM (ICD-9 153-154)}

EUROPE (EC), MORTALITY

Percentage change per five-year period, 1975-1988

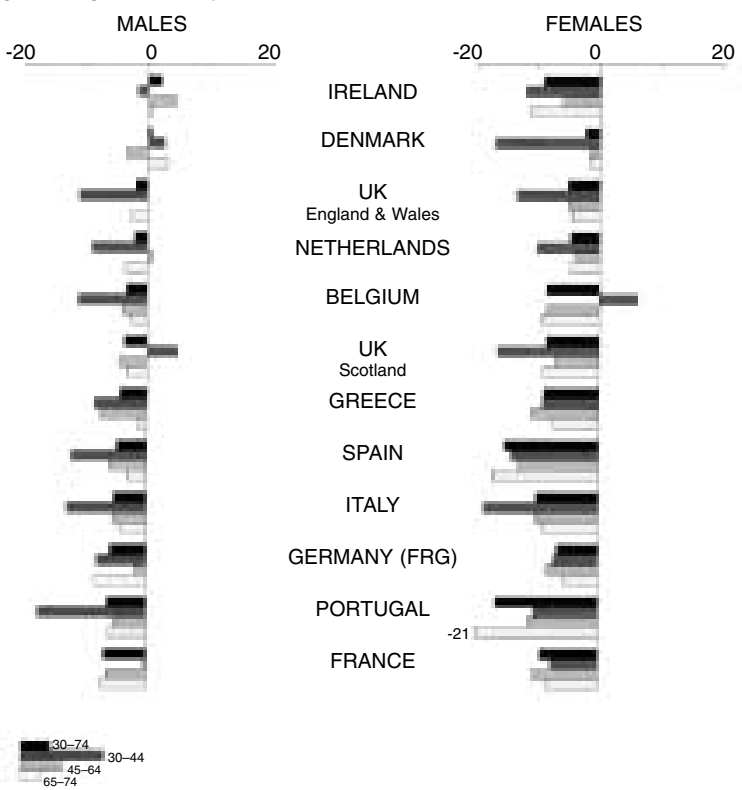

Fig. 6.: Colorectal cancer age-specific mortality rates, estimated mean percentage change per five-year period over the period 1975-1988 - EC countries 


\section{SURVIVAL}

Five-year survival rates are generally about $50 \%$. Data from the EUROCARE study in the period 1978-1985 show that females survived longer than males with rectal cancer (not with colon cancer space). There is a relative five-year survival improvement over time and relative survival was generally better in countries with high life-expectancy and good access to well organized modern specialized care. (fig. 7, 8).

\section{COLON}

RELATIVE SURVIVAL (\%)

(Age-standardized)

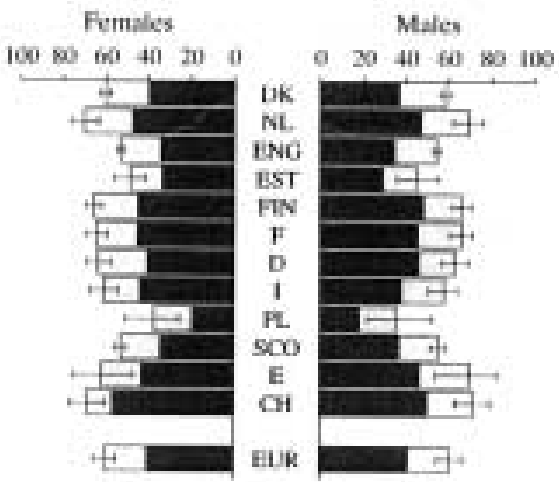

RELATIVE SURVIVAL (\%) BY AGE AND PERIOD ACE
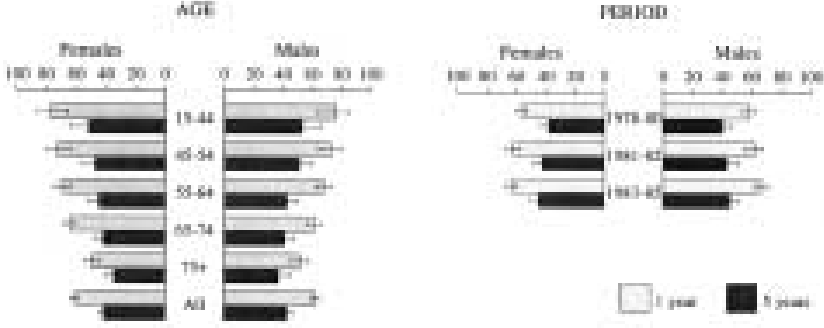

Fig. 7.: Colon cancer age-standardized relative survival

\section{RECTUM}

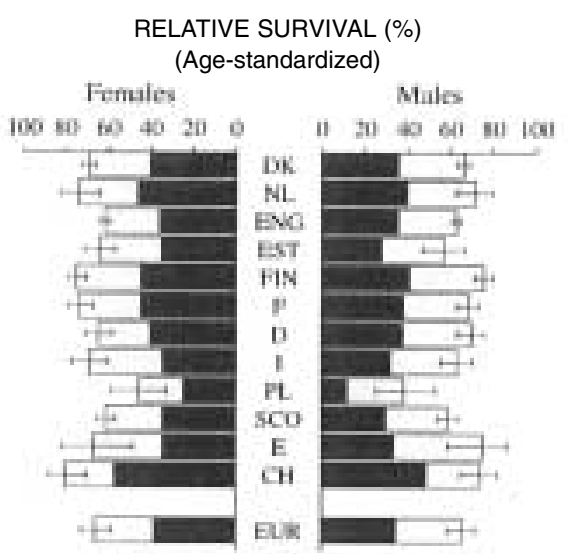

RELATIVE SURVIVAL (\%) BY AGE AND PERIOD

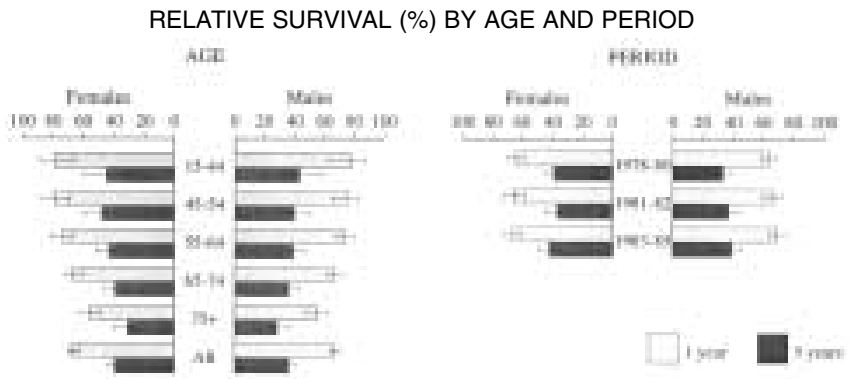

Fig. 8.: Rectal cancer age-standardized relative survival

\section{RISK FACTORS}

Familial and hereditary factors have been observed in the aetiology of colorectal cancer.

In addition to that, a substantial number of environmental and lifestyle risk factors may play an important role in the development of colorectal cancer:

- adenomatous polyp

- inflammatory bowel disease

- dietary factors

- fat and energy

- fiber, fruits, vegetables

- calcium and vitamin D

- alcohol and tobacco

- cholecystectomy

- irradiation.

\section{ADENOMATOUS POLYP}

Neoplastic polyps (namely tubular and villous adenomas) are precursor lesions of colorectal cancer.

Adenomas with high-grade dysplasia are more likely to contain foci of invasive cancer. The malignant potential of an adenoma is predicted by:

- its size ..... $1.0 \mathrm{~cm}$ or larger (surface diameter)

- the presence of high-grade dysplasia

- a predominance of villous features.

The frequency of adenomas in various populations parallels the level of colorectal $\mathrm{Ca}$ incidence.

\section{INFLAMMATORY BOWEL DISEASE}

This regards mainly ulcerative colitis and Crohn's disease. Patients with IBD are at an increased risk and the magnitude of risk increases in relation to:

- early age at diagnosis

- duration of symptoms

- extent of mucosal involvement

- biopsy demonstration of foci of dysplasia in flat mucosa or adenomatous polyp

The overall increase in RR in patients with IBD has been estimated between 4-20 fold.

\section{DIETARY FACTORS}

Dietary macronutrient and micronutrient factors may enhance or inhibit carcinogenesis in the colon and rectum. There is a multistep molecular biology process of colorectal cancer development with regards to the role of diet and the following factors participate in this process:

- mutagens appear naturally in foods or as a result of cooking meat proteins

- conversion of bile acids to tumor-promoting chemicals by normal colonic bacteria which is enhanced by a high fat and low fiber diet

- the formation of oxygen radicals and lipid peroxidation products by diet-induced metabolic activation of procarcinogens. 
Despite that, there is still a general impression of inconsistency and continuing controversy about the role of dietary factors due to the following facts:

- exposure to nutrients may be measured with instruments of uncertain reliability and validity

- dose-response relationships frequently are not compatible with the assumption of linearity

- degree of confounding by other nutrients and total energy consumption is not adequately followed and varies with race, ethnicity and sociocultural factors.

\section{Fat and energy}

In experimental animal studies increasing caloric intake (regardless of nutrient source) potentiated the incidence of tumors induced by a carcinogen.

In epidemiological studies with the association of meat or animal fat with colon cancer, a positive association with total energy intake has also been described and, in many epidemiological studies, a positive association of increased energy intake and colorectal cancer in both men and women has been observed.

The demonstration of an association with total energy intake raises additional questions about :

- the biological importance of energy balance between intake and physical activity

- body composition and distribution of adiposity

- individual variation in metabolic efficiency

- the independent relationship with fat as an energyproviding macronutrient

- other lifestyle factors associated with energy output.

The hypothesis that diets high in animal fat and meats (and low in plant fiber, fruits and vegetables) were a cause of colorectal cancer was partly derived from the correlational studies between per capita consumption of animal fat and age-adjusted colorectal cancer incidence rates $(r=0.9)$

The hypothesis is supported by case-control and cohort studies with the conclusion that fat intake was a significant independent predictor of risk.

But in the prospective Nurses' Health Study neither total energy intake nor BMI were risk predictors. But RR for animal fat consumption was 1.9-2.5.

\section{Animal fat}

There is evidence that a diet rich on animal fat, as is the Western diet, is a major risk factor. The proportion of fat in a Western diet is significantly greater than that in the diet of low-risk populations.

The implication of fat, as a possible etiologic factor, is linked to the concept of the typical Western diet, which favours the development of a bacterial flora capable of degrading bile salts to carcinogens. This diet also seems to lead to a greater excretion of bile salts in the faeces. In this context bacteria within the large bowel play an important role.

\section{Fiber}

The concept of fiber deficiency as an etiologic factor in colorectal cancer has evolved on the basis of compari- sons of patterns of large intestinal diseases in Western and traditional African societies ( a rarity of appendicitis, diverticular disease of the colon, colonic adenomas and carcinomas and IBD was found in rural African populations with high fiber intake as found by Painter and Burkitt in 1971).

An inverse correlation between dietary fiber consumption in different countries and age-adjusted colon cancer mortality rates was also found.

The hypothesis of dietary fiber as a risk factor for colorectal cancer was tested also by IARC in a detailed study of diet, transit time and stool bacterial content. The situation was compared between Kuopio, which is a rural farming area in Finland, and Copenhagen, the capital of Denmark. The incidence rates in Copenhagen were found to be four times those of Kuopio. It was presumed that higher meat intake and beer consumption in Copenhagen was the reason, contrary to higher fiber intake and milk consumption in Kuopio.

Despite supportive evidence there are still criticisms of this concept and other contrary reports that question its validity. In supportive studies the populations differed in various respects other than dietary intake and these factors might have been responsible for the differences in the incidence. Experimental studies also produced conflicting results.

Thus, although the fibre concept appears attractive in theory, there is as yet little scientific evidence to confirm it.

\section{Fruits and vegetables}

Many epidemiological studies, mostly case-control studies, confirmed a significant inverse relationship between colonic cancer and vegetable and fruit consumption.

The consumption of raw or fresh vegetables, especially leafy green vegetables, cruciferous vegetables or specifically broccoli, carrots and raw or fresh fruit, including citrus, as well as allium vegetables (onion, garlic, chive) is emphasized.

The lack of vegetables, in particular those of the cruciferous family such as cabbage, is probably a major dietary cause of colorectal cancer.

Support for this theory also comes from experimental work, because the activity of arylhydrocarbon hydroxylase in the colons of rodents acts under normal circumstances as a metabolic barrier to noxious chemicals and several cruciferous vegetables significantly increase the activity of this enzyme so that active ingredients like indoles inhibite the activity of carcinogens in the induction of gastrointestinal tumors.

The cruciferous vegetables contain a variety of phytochemicals including sulphoraphane, dithiolthiones, isothiocyanates and glucobrassicin a complex alkaloid which yields a variety of indolemethylene compounds, including indole3-carbinol. A metabolite of indole-3-carbinol, 3,3'-diindolylmethane, is an active inducer of cytochrome P450, enzymes of the phase I oxidation, which detoxify carcinogens.

The allium vegetables contain chemical compounds which induce enzymes such as glutathione S-transferase, which are involved in the detoxification of metabolites. 


\section{Calcium and vitamin D}

Calcium depletion might play a part in colon carcinogenesis. Some evidence suggests that bile acids may have a direct toxic action on the colonic mucosa which may lead to neoplastic change. This toxic effect may be related to the amount of calcium present in the diet, so that bile acids bind to it.

Calcium depletion of the mucosa leads to cell desquamation and proliferation and if bile acids remain unbound, they are free to cause alterations in cellular DNA leading to neoplastic change.

\section{Alcohol}

In cohort studies, an association between mortality and alcohol intake was found. In case-control studies results were inconsistent and in correlational studies the association with rectal $\mathrm{Ca}$ was found (fig. 9).

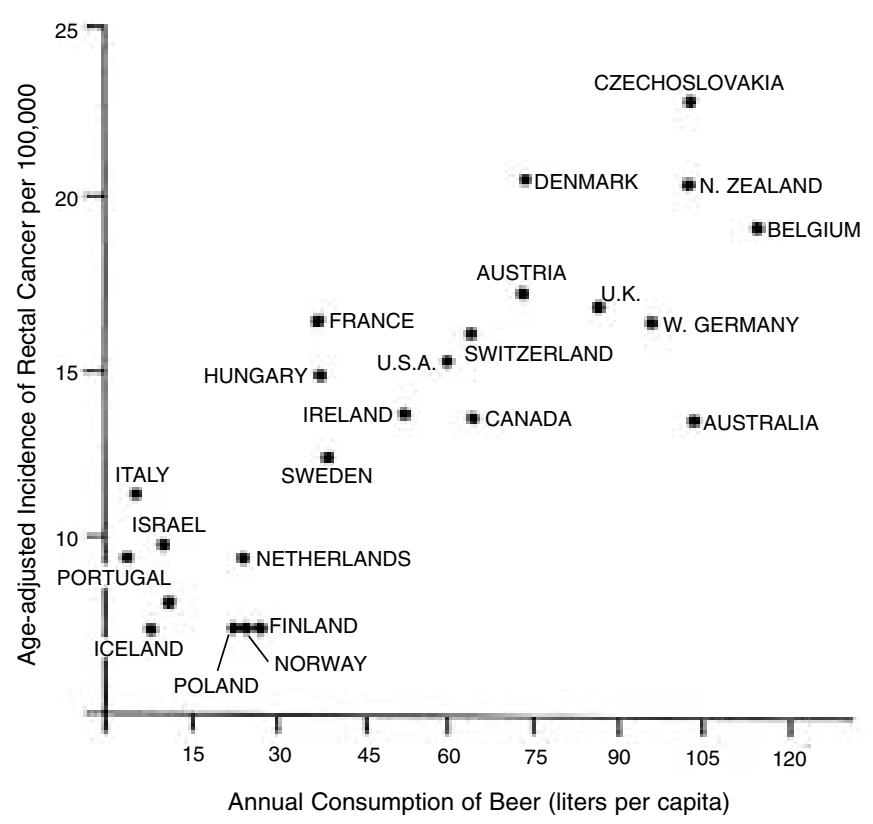

Fig. 9.: Correlational study of the association of alcohol intake and rectal cancer incidence, (Breslow 1974)

The IARC Working Group presented the following summary statement in 1988: ,overall, some of the epidemiological studies provide suggestive but inconclusive data for a causal role of the drinking of alcoholic beverages, most often beer consumption, in rectal cancer".

\section{NUTRITION - SUMMARY}

There are some other nutritional factors which may be associated with the increasing risk of colorectal cancer. The summary of all possible nutritional risk factors include:

- energy intake has no simple relationship

- frequent eating possibly increases the risk

- being tall as an adult possibly increases the risk

- obesity possibly increases risk of colon cancer (men), perhaps not rectal cancer

- diets high in starch possibly decrease the risk (protective effect more likely in unrefined)
- diets high in extrinsic (refined) sugars possibly increase risk (evidence strongest for sucrose)

- diets high in fibre (vegetables, fruits, cereals, pulses, seeds) possibly decrease the risk

- diets high in total fat possibly increase the risk (mainly saturated/animal fat)

- dietary cholesterol may increase the risk

- high alcohol consumption probably increases the risk, the effect seems to be related to total ethanol intake

- red meat, probably, and processed meat possibly increase the risk (it is unclear whether the specific mechanisms involve animal fat, processing and cooking methods or other factors)

- consumption of eggs possibly increases the risk

- cooking meat at high temperatures possibly increases the risk (grilling, barbecuing, frying)

\section{SMOKING}

There is a modest association between smoking and colorectal cancer. A relationship was found between smoking and the risk of adenomatous polyps in the colon and rectum. Causal relationship association is consistent with respect to:

- men and women

- both proximal and distal locations

- small $(<1 \mathrm{~cm})$ and large $(>1 \mathrm{~cm})$ adenomas

- dose response gradients

- induction-latency period of less than 20 years (from the initiation of smoking for small adenomas and at least 20 years for larger)

\section{CHOLECYSTECTOMY}

A hypothesis was established in 1978 , since then more than 60 studies have attempted to confirm it.

A biological mechanism is explained by the continuous secretion of bile into the gut as a result of the absence of the gallbladder.

A meta-analysis of 38 cohort and case-control studies (1993) determined the pooled RR for men at 1.21, for women at 1.24 and for right-sided colon Ca 1.33-1.88.

It may be concluded that cholecystectomy may be associated with a small increase in RR of proximal colon cancer that is perhaps less than $50 \%$.

\section{IRRADIATION}

Patients who have received pelvic irradiation seem to be at higher risk of developing rectosigmoideal cancer.

The following criteria has been established for classifying a tumor as radiation induced:

- adequate time (10 years or more)

- radiation exposure should be considerable

- damage of tissue adjacent to tumor is severe.

The relative risk after irradiation for gynaecological cancer was 2.0-3.6 and the risk after pelvic irradiation for benign conditions was 1.0-8.0. 


\section{PRIMARY PREVENTION}

Here are the most important ideas for primary prevention of colorectal cancer:

1. nutritional changes

2. use of nonsteroidal antiinflammatory pharmaceuticals like aspirin, ibuprofen, indomethacin, piroxicam

3. menopausal replacement oestrogens

4. physical activity

- consistently associated with decreased risk of colon

cancer (it is not as clear for rectal cancer)

- stimulation of colon peristalsis

- decreasing the timeframe for dietary factors

- influence on T and B cells, NK, interleukin-1

5. use of micronutrients (carotenoids, vitamin $\mathrm{C}$, folate and methionine, vitamin $\mathrm{E}$, vitamin $\mathrm{D}$, calcium)

\section{REFERENCES}

1. Cancer Incidence in Five Continents, IARC Scientific Publication No. 143, 1997

2. Trends in Cancer Incidence and Mortality, IARC Scientific Publication No. 121, 1993

3. Survival of Cancer Patients in Europe, IARC Scientific Publication No. 132, 1995

4. Cancer Epidemiology and Prevention, Oxford University Press, 1996

5. Food, Nutrition and the Prevention of Cancer: a global perspective, World Cancer Research Fund, 1997

6. Cancer Epidemiology: Principles and Methods, IARC, 1999

Paper presented at the Training Course on Colorectal Cancer in Prague, Czech Republic, April 13.-14.2000, jointly organized by CECOG (Central European Co-operative Oncology Group) and ESO (European School of Oncology), Vienna Office. 